\title{
The electromagnetic interference caused by high voltage power lines along the electrical railway equipment
}

\author{
Moine EI Hajji, Hassane Mahmoudi, Moussa Labbadi \\ Power Electronics Team, Intelligent Control, Automatic and Robotic Techniques, Department of Electrical Engineering, \\ Mohammadia School of Engineers, Mohammed V University, Morocco
}

\begin{tabular}{l} 
Article Info \\
\hline Article history: \\
Received Nov 8, 2019 \\
Revised Mar 24, 2020 \\
Accepted Apr 5, 2020 \\
\hline Keywords: \\
Electric railway \\
EMC \\
MTL theory \\
Power transmission line \\
Signalization system
\end{tabular}

Signalization system

\begin{abstract}
The identification of risks linked to electromagnetic compatibility (EMC) in the electric railway is a major concern in identifying EMC problems and analyzing the unintentional various external disturbing sources as well as the probability of occurrence of interference, the level of interference along the railway system. The purpose of this analysis is to determine the electromagnetic interaction coupling generated by the high voltage (HV) lines located along the railway line by analyzing the voltage induced in the signaling transmission cables such as the european rail traffic management system/european train control system (ERTMS/ETCS) through the multi-conductor transmission line (MTL) theory which may have an impact on the transmitting information. Dubanton method and approximate calculation will be applied and simulated through COMSOL Multiphysics tool in order to analyze if the protection distance and coupling conditions are respected by the railway standards.
\end{abstract}

Copyright $(2020$ Institute of Advanced Engineering and Science. All rights reserved.

\section{Corresponding Author:}

Moine El Hajji,

Power Electronics Team, Intelligent Control, Automatic and Robotic Techniques,

Department of Electrical Engineering, Mohammadia School of Engineers,

Mohammed V University, Rabat, Morocco.

Email: moine.elhajji@gmail.com

\section{INTRODUCTION}

The electromagnetic environment of the HV lines along the railway lines can be source of interference if field's levels exceed the fields taken into account in the standards fixed by the EN 50121. At low frequencies, HV lines can be sources of very strong magnetic field. They may cause disturbances to devices that are sensitive to magnetic fields. The proximity of the HV pylons can cause an increase in potential in the neighboring structures. This document deals with the electromagnetic compatibility between the railway equipement installed such as the signaling system and the external high voltage lines installed near the railway lines.

The coupling sections are located by determining the zones of the electromagnetic disturbances of the overhead power lines with an order of about $10 \mathrm{~m}$ for capacitive coupling and $2000 \mathrm{~m}$ for inductive coupling. An electromagnetic model of railway signal cables and conductors of the overhead power lines will be modelled to closely understand and analyze the electrical behavior of the system in question through the theory of the multi-conductor transmission lines by calculating mutual admittance and mutual inductance through two analytical methods that are Dubanton and approximate methods. The Dubanton method is in the complex plan and it is characterized as a simple relation for the calculation of the coefficients of the mutual impedance in order to obtain a correct calculation up to $10 \mathrm{kHz}$. However, the approximate method is given by a mathematical approach.

This paper is organized as follows: in section 2, the multiconductor transmission line theory is presented by calculating the inductance and the admittance matrix to analyze the electromagnetic 
interference caused by the overhead electric power lines near the railway signaling lines along the railway track [1]. In section 3, simulation is presented through COMSOL Multiphysics in order to calculate the electrostatic and magnetic coupling disturbances. Then we will finish our study with a conclusion.

\section{MULTICONDUCTOR TREANSMISSION LINE THERY}

\subsection{Presentation of the MTL theory}

The proximity of the HV power lines and the railway installation track can cause induced currents and voltage on the signaling cables located along the railway track. The MTL theoryis used to represent and to analyze the coupling between the disturbed and disturbing installations [2]. Electromagnetic disturbance in our study is an electrical network characterized by magnetic field $\mathrm{H}$ and electric field $\mathrm{E}$. The capacitive and inductive coupling will be studied which are respectively the result of the potential difference between conductors and the result of coupling between conductors when a large current is carried out, and which may generate a high magnetic disturbance around the railway equipement. The model under study which is the high voltage pylon in parallel to the electric railway track is represented in the Figure 1.

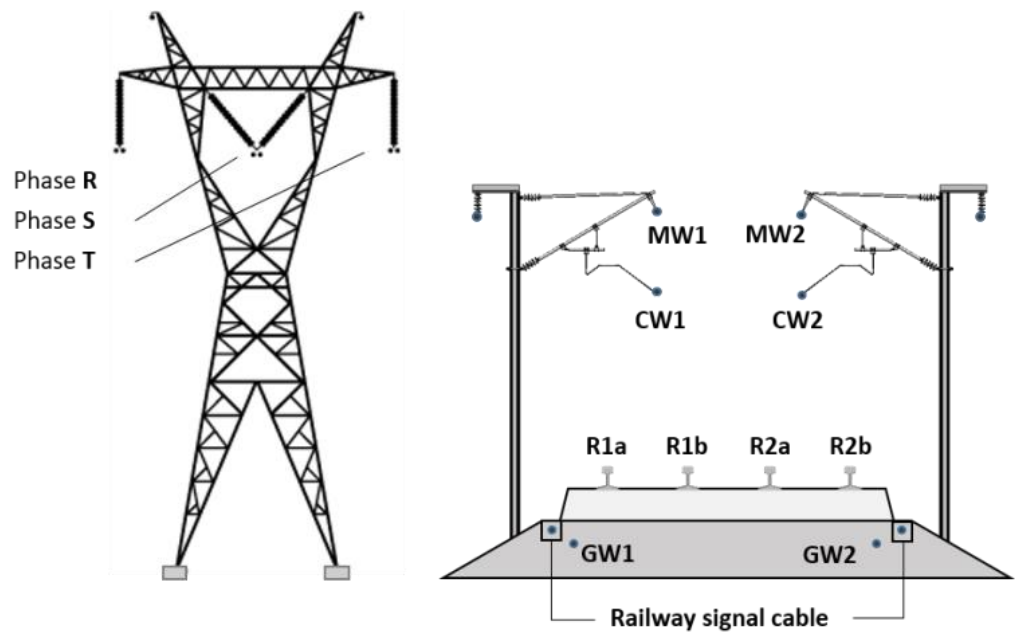

Figure 1. Extra high voltage pylons near the electric railway installation

Note: MW: Messenger Wire,

CW: Contact Wire,

GW: Ground Wire,

R: Rail.

The location of the extra high voltage power lines (EHV) located along the railway line may have a serious impact which can cause induced currents at the signalization cables located along the railway track [3]. There are different power supply voltages that are provided by different power grid providers and also different type of approximation such as crossover, oblique and parallel. The railway system adopted is the $2 \times 25 \mathrm{kV}-50 \mathrm{~Hz}$ concept.

The presence of conductors located on the extra high voltage and their influence into a single conductor which is the signal cables can be simplified and illustrated in the Figure 2. The concept of the overhead lines with the ground return is been considered as a theory in order to analyze the electromagnetic interaction. $\boldsymbol{U}_{\boldsymbol{B} \boldsymbol{Q}}$ and $\boldsymbol{I}_{\boldsymbol{B} \boldsymbol{Q}}$ are respectively the induced voltage and current when EHV metallic pylone is near the electric railway installation. $\boldsymbol{U}_{\boldsymbol{B} \boldsymbol{Q}}$ is the resistive inductive coupling voltage caused by the interference between the other conductors and the single conductor under analyze.

$$
\underline{U_{B Q i}}=\sum_{k=1 ; k \neq i}^{n} \underline{\boldsymbol{Z}_{\boldsymbol{i k}}} \cdot \underline{\boldsymbol{I}_{\boldsymbol{k}}}
$$

To note that $\boldsymbol{Z}_{\boldsymbol{i} \boldsymbol{k}}$ is the impedence between the conductors ' $\mathrm{k}$ ' and ' $\mathrm{i}$ '. 
$\boldsymbol{I}_{\boldsymbol{k}}$ is the current in the conductor $\mathrm{k}$.

$\overline{\boldsymbol{I}}_{\boldsymbol{B} \boldsymbol{Q}}$ is the the capacitive coupling current caused by the interference between the other conductors and $\overline{\text { the }}$ single conductor under analyze.

$$
\underline{I_{B Q i}}=\sum_{k=1 ; k \neq i}^{n} j w \boldsymbol{C}_{\boldsymbol{i} \boldsymbol{k}} \cdot \underline{\boldsymbol{U}_{\boldsymbol{k}}}
$$

$\boldsymbol{C}_{\boldsymbol{i} \boldsymbol{k}}$ is the capacitance between ' $\mathrm{k}$ ' and ' $\mathrm{i}$ ' conductors.

$\boldsymbol{U}_{\boldsymbol{k}}$ is the voltage between the phase and the ground of the ' $\mathrm{k}$ ' conductor.

$\overline{\text { To }}$ calculate the induced current, we will consider the grounding of the two conductors ends:

$$
\underline{I_{I C}}=\frac{U_{B Q i}}{\overline{Z_{i i}}}
$$

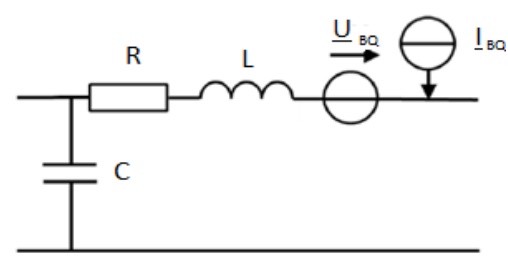

Figure 2. A front view of the railway track

The electromagnetic field is divided into two components, the magnetic field and the electric field. Both fields will be analyzed separately in normal conditions and in different time instants. The equivalent electrical circuit is represented in Figure 3. It is illustrated through four wire transmission lines (railway signal cable, 3-phase power line) with $\mathbf{Z}_{\mathbf{i j}}$ mutual impedances, $\mathbf{Z}_{\mathbf{i i}}$ impedances, $\mathbf{Y}_{\mathbf{i j}}$ mutual admittances and $\mathbf{Y}_{\mathbf{i i}}$ admittances. The model of the MTL theory [4] under study is represented in the Figure 4.

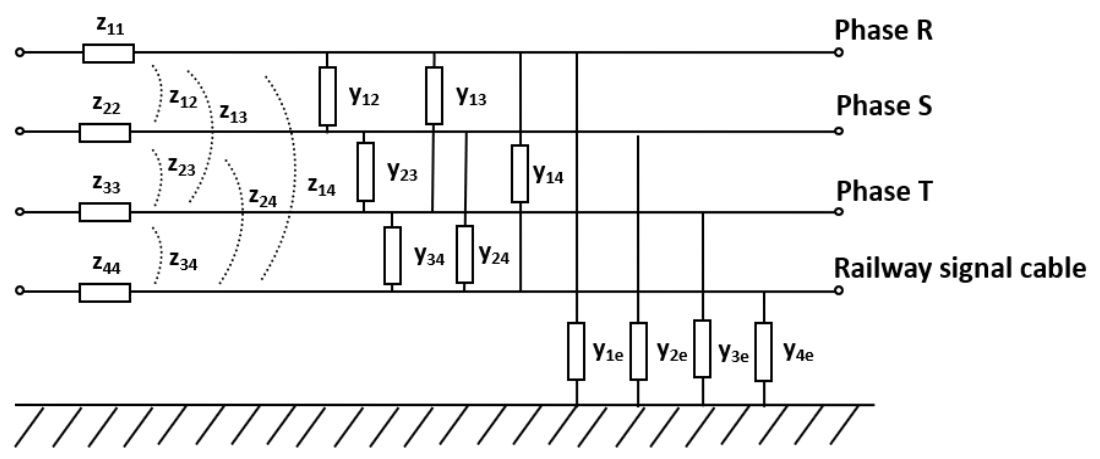

Figure 3. Transmission lines (4 wires: phase R, S, T, railway signal cable)

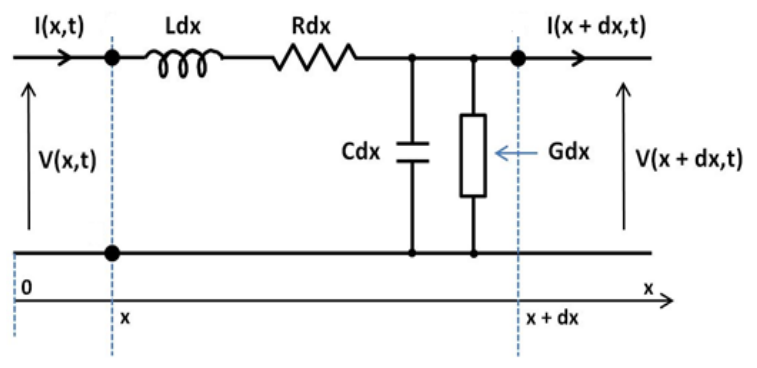

Figure 4. The multiconductor transmission line model 
The differential equations are written for the transmission line for each parameter line voltage and current:

$$
\left\{\begin{array}{l}
\frac{\partial V(x, t)}{\partial x}=-L \frac{\partial I(x, t)}{\partial t}-R I(x, t) \\
\frac{\partial I(x, t)}{\partial x}=-C \frac{\partial V(x, t)}{\partial t}-G V(x, t)
\end{array}\right.
$$

In order to simplify the model under study, the voltage along one phase is given by the following relation:

$$
\frac{d V_{1}}{d x}=-Z_{11} I_{1}-Z_{12} I_{2}-Z_{13} I_{3}-Z_{14} I_{4}
$$

with, $Z_{11}$ is the impedance of one phase (phase R),

$$
Z_{11}=R_{11}+j w L_{11}
$$

and $Z_{1 j}$ is the mutual impedance between the $\mathrm{j}$ lines and the phase $\mathrm{R}$.

$$
Z_{1 j}=R_{1 j}+j w L_{1 j}
$$

In a matrix format:

$$
\frac{d[V]}{d x}=-[Z][I]
$$

The impedance matrix $[Z]$ is given below:

$$
[Z]=\left[\begin{array}{llll}
Z_{11} & Z_{12} & Z_{13} & Z_{14} \\
Z_{21} & Z_{22} & Z_{23} & Z_{24} \\
Z_{31} & Z_{32} & Z_{33} & Z_{34} \\
Z_{41} & Z_{42} & Z_{43} & Z_{44}
\end{array}\right]
$$

The coefficients $Z_{\mathrm{ii}}$ is the impedance of the line "i" and per unit length and the coefficients $Z_{\mathrm{ij}}$ and to the ground which represent the mutual impedance between the "i", "j" lines related to the ground [5]. Current and Voltage vectors are given below:

$$
[V]=\left[\begin{array}{l}
V_{1} \\
V_{2} \\
V_{3} \\
V_{4}
\end{array}\right],[I]=\left[\begin{array}{l}
I_{1} \\
I_{2} \\
I_{3} \\
I_{4}
\end{array}\right]
$$

The current along the "i" line is written by the following relation:

$$
\frac{d I_{i}}{d x}=-y_{i} V_{i}-\sum_{j=1}^{4} y_{i j}\left(V_{i}-V_{j}\right), i \neq j
$$

with, $y_{i j}$ is the mutual admittance between the line " $\mathrm{i}$ " and the line " $\mathrm{j}$ " related to the ground and $y_{i}$ is the admittance of the "i" line.

Matrix form is represented by the following equation:

$$
\frac{d[I]}{d x}=-[Y][V]
$$

The admittance matrix $[Y]$ is given below:

$$
[Y]=\left[\begin{array}{llll}
y_{11} & y_{12} & y_{13} & y_{14} \\
y_{21} & y_{22} & y_{23} & y_{24} \\
y_{31} & y_{32} & y_{33} & y_{34} \\
y_{41} & y_{42} & y_{43} & y_{44}
\end{array}\right]
$$


The coefficients $y_{\mathrm{ij}}$ is the admittances between the " $\mathrm{i}$ " and " $\mathrm{j}$ " lines related to the ground and $y_{\mathrm{ii}}$ is the admittances per unit length [6]. The direct equivalence between the coefficients of the physical admittances and the admittance matrix does not exist. The coefficients of the admittance matrix for only one transmission line are given by the following equations:

$$
\left\{\begin{array}{c}
y_{i i}=y_{i}+\sum_{j=1}^{4} y_{i j}, i \neq j \\
y_{i j}=-y_{i j}
\end{array}\right.
$$

All the conductors are represented of the power transmission lines and the railway signaling cable as a multiconductor transmission which is an approximate approach when the values of the coefficients of admittances and impedancesmatrix are known. The values of the equivalent components are difficult to measure under practical conditions, because they depend on the parameters of the cables such as the position, shape, conductivity and permeability, and the conductivity and permittivity of the ground.

\subsection{Coefficients of the impedance matrix calculated}

The impedance of each transmission line and the ground depends mainly on the external impedance [7], the internal impedance of the conductor and the impedance of the ground are given by the following equation:

$$
z_{i i}=z_{\text {int }}+z_{g}+j w l_{s}
$$

with, $z_{\text {int }}$ represent the internal impedance, $z_{g}$ represent the impedance of the ground of the conductor. with, $l_{s}$ represent the inductance per unit length calculated with a perfect conducting ground.

$$
l_{s}=\frac{\mu_{0}}{2 \pi} \ln \left(\frac{2 h}{a}\right)
$$

with $h$ is considered to be the height between the ground and the conductor line and $a$ is the radius of the conductor line.

The internal impedance $z_{\text {int }}$ of the conductor is represented by the following equation:

$$
\begin{aligned}
& z_{\text {int }}=\frac{1}{2} R_{d} \cdot m \cdot a \frac{\text { berm } \cdot a+j \text { beim } \cdot a}{\text { ber }^{\prime} m \cdot a+j \text { bei' }^{\prime} \cdot a} \\
& m=\sqrt{w \cdot \mu_{c} \cdot \sigma_{c}}
\end{aligned}
$$

with $\sigma_{c}$ is the conductivity, $R_{d}$ is the resistance and $\mu_{c}$ is the permability.

And ber, bei represent Kelvin functions, ber' and bei' represent their derivatives.

In order to have a valid result in an extended frequency band, the impedance of the ground $z_{g}$ is represented by the Carson formula:

$$
z_{g}=j w \frac{\mu_{0}}{\pi} \int_{0}^{\infty} \frac{e^{-2 h \lambda} \cdot \cos (\lambda a)}{\lambda+\sqrt{\lambda^{2}-\gamma_{g}^{2}}} \cdot d \lambda
$$

$\gamma_{g}$ is the ground propagation constant defined by the formula:

$$
\gamma_{g}=\sqrt{j w \mu_{0}\left(\sigma_{g}+j w \varepsilon_{0} \varepsilon_{r}\right)}
$$

with, $\mu_{0}$ represent the permeability of the ground, $\varepsilon_{r}$ represent the relative permittivity, $\varepsilon_{0}$ represent the permittivity of vaccum and $\sigma_{g}$ represent the conductivity of the ground.

Mathematical equations of Dubanton's method is used in this study to calculate the parameters of the power transmission lines [8]. The eigen coefficients of the impedance matrix $z_{i i}$ is given by the following formula: 


$$
z_{i i}=z_{\text {int }}+j w \cdot \frac{\mu_{0}}{\pi} \ln \left(\frac{2\left(h_{i}+P\right)}{a}\right)
$$

The mutual coefficients $z_{i j}$ of the impedance matrix is given otherwise through the following formula:

$$
\begin{aligned}
& z_{i j}=j w \frac{\mu_{0}}{\pi}\left(\frac{1}{2} \ln \left(\sqrt{\frac{\left(h_{i}+h_{j}\right)^{2}+d^{2}}{\left(h_{i}-h_{j}\right)^{2}+d^{2}}}\right)+\int_{0}^{\infty} \frac{e^{-2 h \lambda} \cdot \cos (\lambda a)}{\lambda+\sqrt{\lambda^{2}-\gamma_{g}^{2}}} \cdot d \lambda\right) \\
& =>z_{i j}=j w \frac{\mu_{0}}{2 \pi} \ln \left(\sqrt{\frac{\left(h_{i}+h_{j}+2 P\right)^{2}+x_{i j}^{2}}{\left(h_{i}-h_{j}\right)^{2}+x_{i j}^{2}}}\right)
\end{aligned}
$$

The complex depth is $\mathrm{P}$,

$$
P=\sqrt{\frac{\rho}{w \mu_{0}}}
$$

$d$ is the distance between the "i", " $\mathrm{j}$ " conductors, $h_{i}, h_{j}$ are the heights respectively of "i", " $\mathrm{j}$ " conductors, $\rho$ is the soil resistivity, the pulsation of the inductor current $w=2 \pi f$.

$x_{i j}$ is the horizontal spacing between the two conductors, given by the following relation:

$$
x_{i j}=\sqrt{d^{2}-\left(h_{i}-h_{j}\right)^{2}}
$$

\subsection{The coefficients of the admittance matrix calculated}

The admittance matrix for the high voltage power lines is given below:

$$
[Y]=j w[P]^{-1}
$$

$[P]$ is the potential matrix.

$$
[P]=\left[\begin{array}{llll}
p_{11} & p_{12} & p_{13} & p_{14} \\
p_{21} & p_{22} & p_{23} & p_{24} \\
p_{31} & p_{32} & p_{33} & p_{34} \\
p_{41} & p_{42} & p_{43} & p_{44}
\end{array}\right]
$$

The eigen coefficients $p_{i i}$ of the matrix $[\mathrm{P}]$ are given by the following relation:

$$
p_{i i}=\frac{1}{2 \pi \varepsilon_{0}} \cdot\left(\ln \left(\frac{2 h}{a}\right)+\int_{0}^{\infty} 2 k_{0}^{2} \cdot \frac{e^{-2 h \lambda} \cdot \cos (\lambda a)}{\lambda \cdot \gamma_{g}^{2}+k_{0}^{2} \sqrt{\lambda^{2}-\gamma_{g}^{2}}} \cdot d \lambda\right)
$$

$p_{i j}$ mutual coefficients of the matrix [P] of the two conductors related to the ground are given by the following relation:

$$
p_{i j}=\frac{1}{2 \pi \varepsilon_{0}} \cdot\left(\ln \left(\sqrt{\frac{\left(h_{i}+h_{j}\right)^{2}+d^{2}}{\left(h_{i}-h_{j}\right)^{2}+d^{2}}}\right)+\int_{0}^{\infty} 2 k_{0}^{2} \cdot \frac{e^{-\left(h_{i}+h_{j}\right) \lambda} \cdot \cos (\lambda d)}{\lambda \cdot \gamma_{g}^{2}+k_{0}^{2} \sqrt{\lambda^{2}-\gamma_{g}^{2}}} \cdot d \lambda\right)
$$

with,

$$
k_{0}^{2}=\mu_{0} \varepsilon_{0} w^{2}
$$




\section{ELECTROMAGNETIC INTERFERENCES CAUSED BY THE OVERHEAD POWER LINES}

The Dubanton method previously described associated to the theory of MTL has been applied in order to have a better approach which is an analytical calculation simplified with mathematical equations. The electromagnetic field is divided into two components, the magnetic field and the electrostatic field [9]. Both fields will be analyzed separately in normal conditions and in different time instants.

\subsection{Magnetic field}

The magnetic coupling phenomenon caused by the impact between the high voltage power line and the signaling cables is described in the Figure 5.

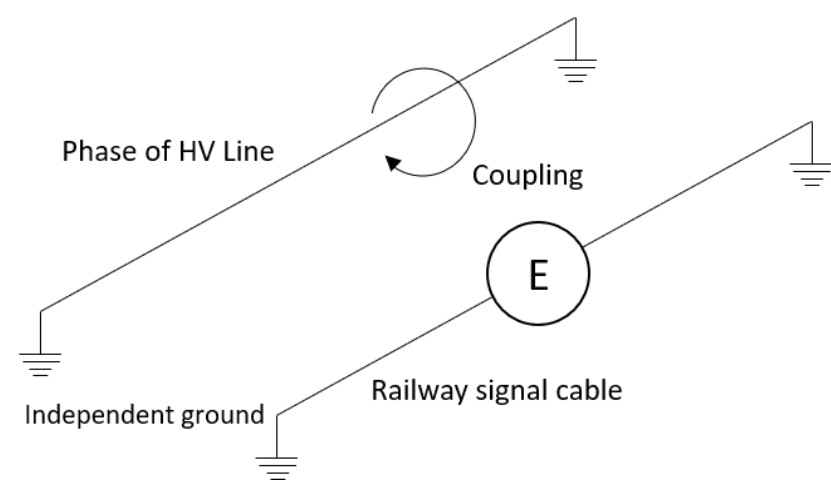

Figure 5. Magnetic induction coupling

The expression of the electromotive force induced $\mathrm{E}$ along each transmission line is given in the form of the sum of all the electromotive forces induced in each section $e_{i j}$. The magnetic induction will be calculated through the approximate formula and the dubanton method.

$$
E=\sum_{i, j} e_{i j}
$$

The Dubanton aproach already expressed in the previous section is given through the following formula:

$$
z_{i j}=j w \frac{\mu_{0}}{2 \pi} \ln \left(\sqrt{\frac{\left(h_{i}+h_{j}+2 P\right)^{2}+x_{i j}^{2}}{\left(h_{i}-h_{j}\right)^{2}+x_{i j}^{2}}}\right)
$$

However, the approximate method is characterized by the following formula:

$$
z_{i j}=j w M
$$

with, $\mathrm{M}$ represented in $\mu \mathrm{H} / \mathrm{km}$, depends mainly on the value of $\mathrm{x}$ and it is equal to:

- For $x \leq 10$ :

$$
M=142+46 x-198 \ln (x)-1.4 x^{2}
$$

- $\quad$ For $x>10$ :

$$
M=400 x^{-2}
$$

with,

$$
x=d \sqrt{\frac{w \mu_{0}}{\rho}}
$$


The magnetic field varies with the variations of the current flowing in the cables. It depends mainly on the current flowing in the nearby conductor transmission lines and their geometry parameters.

In the case of our study, the magnetic field is determined by the following relation:

$$
B=\frac{\sqrt{6} d_{p p} I}{10 r^{2}}
$$

with, $I$ is the current flowing in the high voltage power transmission line, $d_{p p}$ is the distance between the overhead phases, $r$ is the distance of the field relative to the source.

\subsection{Electrostatic field}

Capacitive couplings are generated when two conductor lines are above the ground. Variable electric field are created because of the variable voltage flowing in the conductor as seen in the Figure 6 . Which causes induced currents and voltages on overhead power transmission lines placed along the Catenary lines $2 \times 25 \mathrm{kV}$ system $[10,11]$

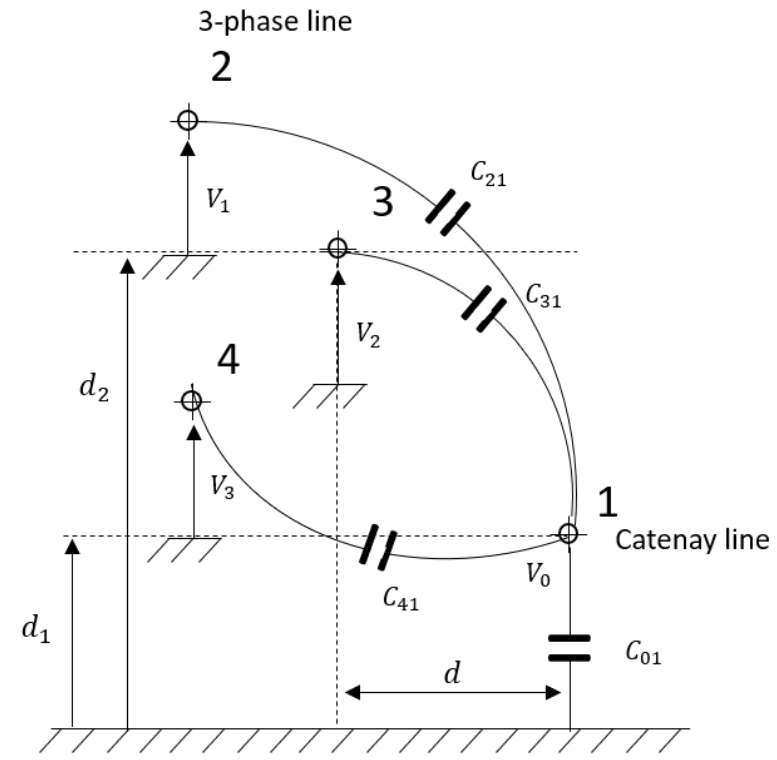

Figure 6. Capacitive coupling generated between the catenary and the 3 phase lines

Capacitive coupling between the signal cables and the extra high voltage lines is considered to be negligible in our study since they are buried under the ground. The catenary line is the only system that is susceptible to be charged with electricity.

$$
V_{0}=\frac{Q_{0}}{C_{01}}=\frac{C_{21} V_{1}+C_{31} V_{2}+C_{41} V_{3}}{C_{21}+C_{31}+C_{41}}
$$

The induced current $I_{0}$ flowing in the conductor is proportional to the capacitive coupling $C_{01}$ between the ground and the catenary line, the length of parallelism 1 andthe induced voltage $V_{0}$.

$$
I_{0}=V_{0} \cdot C_{01} \cdot w \cdot l
$$

\subsection{Results compared to international standard limits}

The magnetic and electric fields are modeled by COMSOL Multiphysics as shown in the Figures 7 and 8 . The values of the parameters in the worst case located along the electric railway track are listed in the Table 1 in order to calculate according to the two methods the mutual inductance listed in the Table 2 to analyze the electric and magnetic field generated. Results are obtained after calculation of the mutual inductance, it is noted that the results of the two methods are close. 


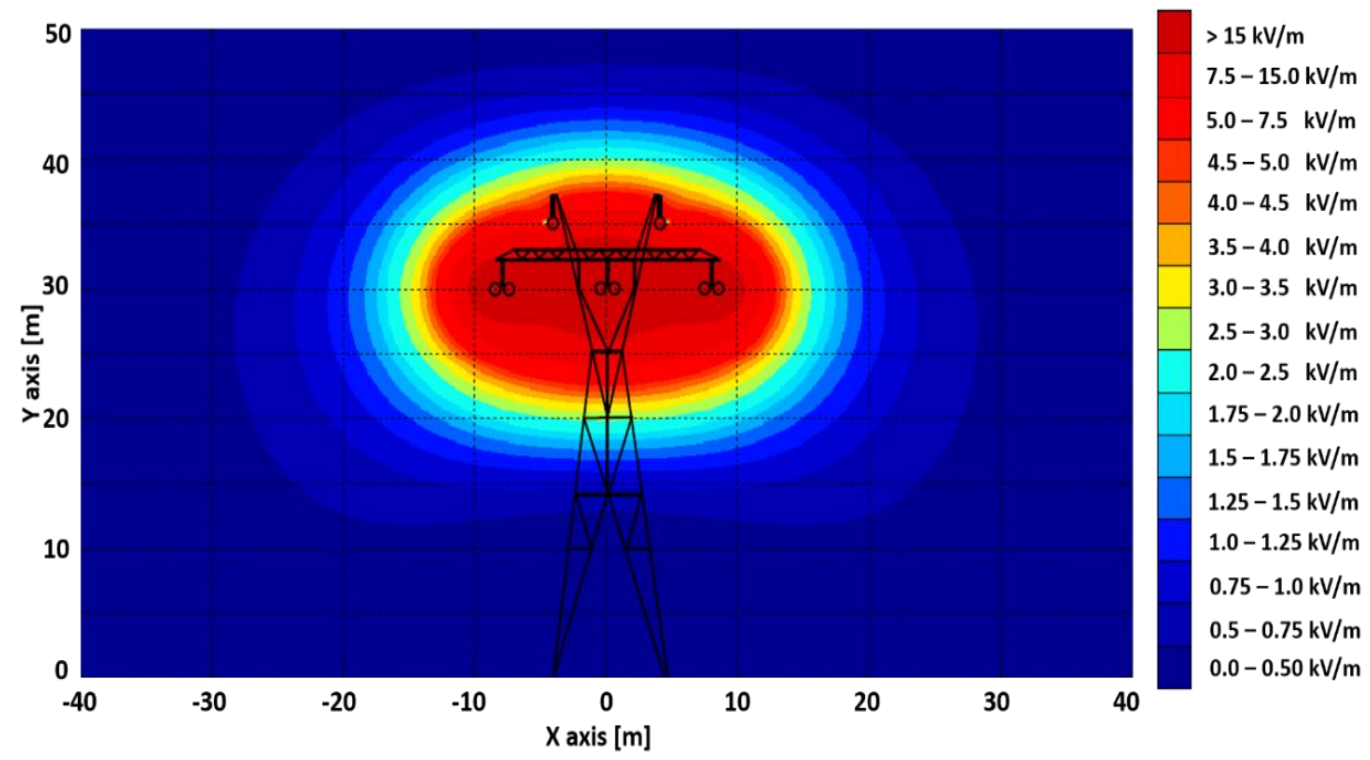

Figure 7. Electric field modelled by COMSOL multiphysics

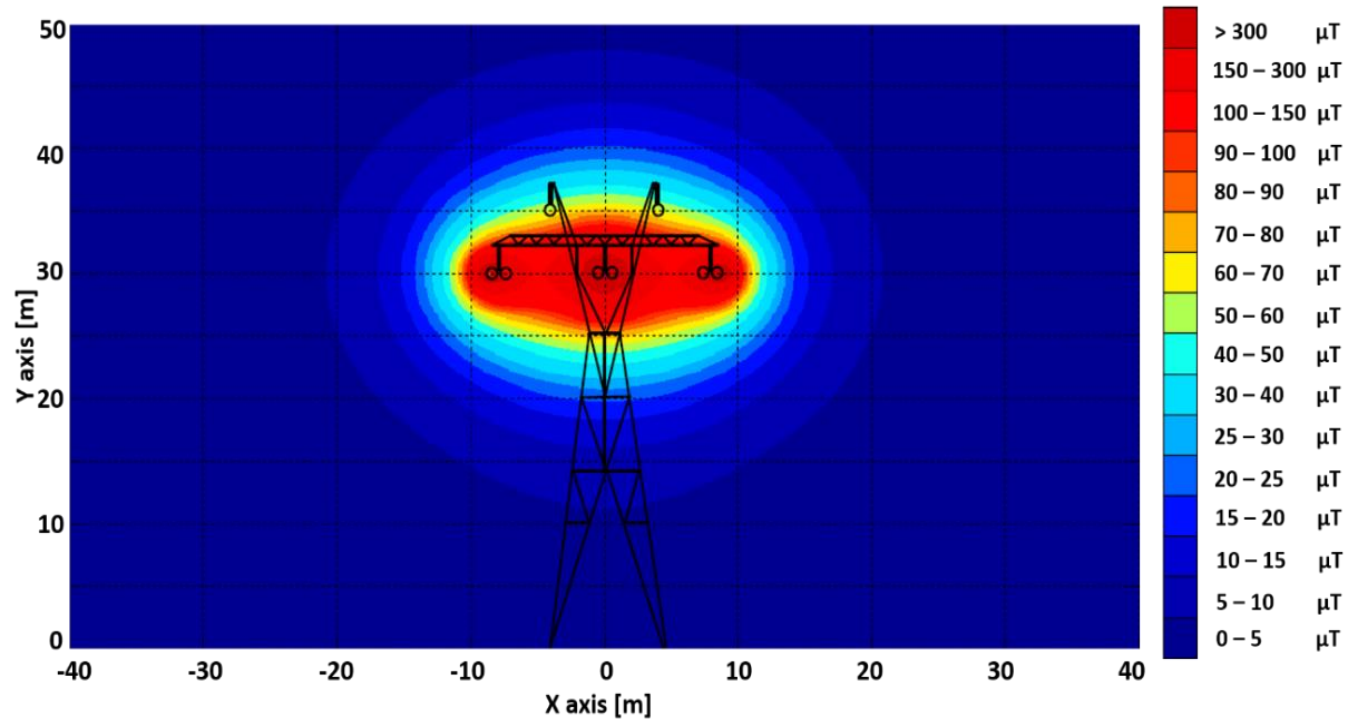

Figure 8. Magnetic field modelled by COMSOL multiphysics

Table 1. Fixed values

\begin{tabular}{ccc}
\hline Variable & Value & Unit \\
\hline$U_{n}$ & 440 & $\mathrm{kV}$ \\
$\rho$ & 280 & $\Omega . \mathrm{m}$ \\
$\mathrm{f}$ & 50 & $\mathrm{~Hz}$ \\
$h_{i}$ & 10 & $\mathrm{~m}$ \\
$h_{j}$ & 10 & $\mathrm{~m}$ \\
$l$ & 1.5 & $\mathrm{~km}$ \\
$d$ & 15 & $\mathrm{~m}$ \\
$d_{1}$ & 5 & $\mathrm{~m}$ \\
$d_{2}$ & 20 & $\mathrm{~m}$ \\
\hline
\end{tabular}

Table 2. Compared values of the mutual inductance

\begin{tabular}{lll}
\hline Formula & Value & Unit \\
\hline Dubanton & 2.346 & $\mu \mathrm{H} \cdot m^{-1}$ \\
Approximate & 2.507 & $\mu \mathrm{H} . m^{-1}$ \\
\hline
\end{tabular}


The capacitive coupling between the signaling cables and the extra high voltage power lines for our study will be negligible because the cables specifications are shielded and according to the transfer of impedance of these cables and the result of the values settled in the worst case is inferior below the required limits fixed by the CENELEC international standards, the internal induced voltages are mitigated. Induced current and voltage flowing the catenary are given by the following formula:

$$
\begin{aligned}
& V_{0}=6,8 \mathrm{~V} \\
& I_{0}=0,1109 \mathrm{~mA}
\end{aligned}
$$

The magnetic field:

$$
B=3,8 \cdot 10^{-5}
$$

The limits are given below for signaling equipment located within $3 \mathrm{~m}$ of the trackaccording to the international standard EN 61000-4-8 EN 50121-4 [12-25]:

- For AC system:

$$
B=\mu_{0} H=3,8 \cdot 10^{-4}
$$

- For DC system:

$$
B=\mu_{0} H=1,25 \cdot 10^{-4}
$$

It can be seen that the results obtained are within the limit range thresholds fixed by the international standardization. Electromagnetic disturbances due to the presence of the extra high voltage pylons along the electric railway lines can be supported by signaling equipments.

\section{CONCLUSION}

EHV power transmission lines are a subject of the electromagnetic interference generated by the magnetic and electric fields. The operation of the installed railway catenary can be disturbed by the EMC problems caused by the proximity and the interaction between EHV pylone and railway catenary. In the present work, electromagnetic modelization and simulation was used in order to analyze the combined effect of electric field and magnetic induction caused by the EHV power transmission line. This modelling methodology is based on the mutliconductrice transmission line theory, the induced voltage and current should not exceed the thresholds recommended by the international standard EN 50121. The results confirm that there is a permanent radiated impact when it comes to having an EHV power line next to the railway system. However, according to the calculation done in this study, there is no impact which may cause a dammage on the functioning of the railway signaling devices.

\section{REFERENCES}

[1] A. Novitskiy, I. Konotop and D. Westermann, "EMC Problems caused by combinations of EHV transmission lines and electrified railway lines," International Conference on Renewable Energies and Power Quality, 2014.

[2] Z. Mazloom, "Multi-conductor transmission line model for electrified railways: a method for including responses of lumped devices," Digitala Vetenskapliga Arkivet, vol. 3, pp. 29-44, 2010.

[3] K. Jia, "Electromagnetic noise generated in the electrified railway propulsion system," Licentiate Thesis Electromagnetic Engineering, Royal Institute of Technology (KTH), School of Electrical Engineering, Stockholm, Sweden, vol. 4, pp. 29-30, 2011.

[4] Adesegun A. Ogunsola, "Railway interference management: TLM modelling in railway applications," Loughborough University, vol. 4, pp. 66-100, 2008.

[5] A. Dolara, M. Gualdoni and S. Leva, "EMC disturbances on Track Circuits in the $2 \times 25 \mathrm{kV}$ high speed AC railway systems," IEEE Trondheim Power Tech, pp. 1-9, 2011.

[6] M. Alejandra, "Contribution à la modélisation CEM en milieu ferroviaire: Influence de l'infrastructure," theses.fr, 2010.

[7] S. Minucci, M. Pagano and D. Proto, "Model of the $2 \times 25 \mathrm{kV}$ high speed railway supply system taking into account the soil-air interface," International Journal of Electrical Power and Energy Systems, vol. 95, pp. 644-652, 2018,

[8] L. Xiaotian, Z. Haijing, Q. Bo and H. Bochong, "EMC in rail transport," Energy Procedia, vol. 104, pp. 526-531, 2016. 
[9] G. Romero and V. Deniau, "Impact of the measurement methods on the characterization of transient electromagnetic (EM) interferences above $2 \mathrm{GHz}$ in a railway environment," URSI AP-RASC, 2019.

[10] L. Liudvinavcius, "Compensation of reactive power of AC catenary," Procedia Engineering, vol. 187, pp. 185-197, 2017.

[11] M. El Hajji, H. Mahmoudi and M. El Azzaoui, "Harmonic analysis caused by static converters of the railway high speed train and their impact on the track circuit," International Conference on Wireless Technologies, Embedded and Intelligent Systems, 2019.

[12] CENELEC EN 61000-4-5, "Electromagnetic Compatibility - part 5: Testing and measurements techniques section 5: surge immunity test," Nov. 2006.

[13] CENELEC EN 61000-4-6, "Electromagnetic Compatibility - part 6: Testing and measurements techniques -5: surge immunity to conducted disturbancesn induced by radio frequency fields," Aug. 2007.

[14] CENELEC EN 61000-4-8, "Electromagnetic Compatibility - part 4: Testing and measurements techniques section 8: Power frequency magnetic field immunity test," Sep. 1993.

[15] CENELEC EN 61000-4-9, "Electromagnetic Compatibility - part 4: Testing and measurements techniques section 9: Pulse magnetic field immunity test," Sep. 1993.

[16] CENELEC EN 61000-4-10, "Electromagnetic Compatibility - part 4: Testing and measurements techniques section 10: damped oscillatory magnetic field immunity test," Sep.1993.

[17] CENELEC EN 61000-4-11, "Electromagnetic Compatibility - part 4: Testing and measurements techniques section 11: Voltage dips, short interruptions and voltage variations immunity tests," Aug. 2004.

[18] CENELEC EN 61000-4-12, "Electromagnetic Compatibility - part 4: Testing and measurements techniques section 12: Ring wave immunity tests," Dec. 2006.

[19] CENELEC EN 61000-4-13, "Electromagnetic Compatibility - part 4: Testing and measurements techniques section 13: Harmonics and inter-harmonics including mains signalingat ac power port, low frequency immunity tests," Jun. 2002.

[20] CENELEC EN 50121-1, “Railway Application: Electromagnetic Compatibility, Part 1: General,” Jul. 2006.

[21] CENELEC EN 50121-2, "Railway Application: Electromagnetic Compatibility, Part 2: Emission of the whole railway system to the outside world," Jul. 2006.

[22] CENELEC EN 50121-3-1, "Railway Application: Electromagnetic Compatibility, Part 3-1: Train and complete vehicle," Jul. 2006.

[23] CENELEC EN 50121-3-2, "Railway Application: Electromagnetic Compatibility, Part 3-2: Rolling Stock Apparatus," Jul. 2006

[24] CENELEC EN 50121-4, "Railway Application: Electromagnetic Compatibility, Emission and Immunity of signaling and telecommunication appliances," Jul. 2006.

[25] CENELEC EN 50121-1, "Railway Application: Electromagnetic Compatibility, Part 5: Emission and immunity of railway fixed power supply installations,” Jul. 2006. 